\title{
A Category of Control Systems
}

\author{
Rory Biggs and Claudiu C. Remsing
}

\begin{abstract}
We construct the concrete category LiCS of left-invariant control systems (on Lie groups) and point out some very basic properties. Morphisms in this category are examined briefly. Also, covering control systems are introduced and organized into a (comma) category associated with LiCS.
\end{abstract}

\section{Introduction}

Category theory provides a convenient framework for studying various problems in systems and control theory. Such an approach has been proved useful, for instance, in the study of quotients of nonlinear control systems [17], bisimulations (for dynamical, control and hybrid systems) [5], [16] as well as mechanical control systems [9]. Various aspects of the problem of reduction of control affine systems (like factorisation, equivalence and classification) were investigated by Elkin [3] (within an appropriate category AS). Other concrete categories (of nonlinear control systems), like Con, CAS, ACCS or ACon $\mathbf{A}_{l}$, have been constructed and studied by several authors in the last decade or so (see, e.g., [16], [17], [9], [15]).

In this paper we shall construct the concrete category LiCS of left-invariant control systems evolving on (real, finite-dimensional) Lie groups. Morphisms in this category are transformations relating trajectories of one system to another. We investigate which transformations propagate trajectories, as well as the relationship between such transformations and Lie group homomorphisms.

Key Words: Control systems category, left-invariant control system, covering system. 2010 Mathematics Subject Classification: Primary 93A10; Secondary 93C10, 93B17.

Received: April, 2011.

Accepted: February, 2012. 
It turns out that a subclass of such transformations can be effectively characterised by dynamics-preserving Lie algebra homomorphisms. Furthermore, (covering systems and) covering morphisms are naturally introduced to relate systems with similar dynamics on different Lie groups, but with the same Lie algebra. (These are particularly useful when the Lie group under consideration has no matrix representation.)

The paper is organized as follows. In section 2 we review some basic facts regarding left-invariant control systems and associated structures such as trajectories, attainable sets and orbits. In section 3 we construct the category LiCS of left-invariant control systems and then examine briefly the (special) morphisms in this category. In section 4 we extend the notion of a covering of a Lie group to a covering of a LiCS-object. Also, we introduce a universal covering system and show that the covering morphisms preserve the attainable set. A few remarks conclude the paper.

\section{Invariant control systems}

Control systems come in many flavours. We take the view that a (smooth) control system is given by a (smooth) dynamical polysystem $(\mathrm{M}, \mathcal{X})$, together with a class $\mathcal{U}$ of "admissible inputs" (cf. [14]). Here, the state space $M$ is a (smooth) manifold, the dynamics (or dynamical law) $\mathcal{X}=\left(X_{u}\right)_{u \in U}$ consists of (smooth) vector fields on $\mathrm{M}$, and the admissible controls $u(\cdot): I \rightarrow U$ may be (Lebesgue) measurable or piecewise constant, or of some regularity type between these two possibilities (on some interval $I \subseteq \mathbb{R}$ ). The control set (or input space) $U$ is usually equipped with a separable metric space structure. (This assumption includes the frequently occurring cases where $U=\mathbb{R}^{\ell}$ or where $U$ is a compact convex subset of $\mathbb{R}^{\ell}$.)

Invariant control systems on Lie groups were first considered in 1972 by Brockett [2] and by Jurdjevic and Sussmann [7]. A left-invariant control system is a control system evolving on some (real, finite-dimensional) Lie group G, whose dynamics $\Xi: \mathrm{G} \times U \rightarrow T \mathrm{G}$ is invariant under left translations. (The tangent bundle $T G$ can be trivialized by left translations; hence $T G$ will be identified with $\mathrm{G} \times \mathfrak{g}$, where $\mathfrak{g}=T_{\mathbf{1}} \mathrm{G}$ denotes the associated Lie algebra.) Such a control system is described as follows (cf. [6], [1], [13], [12])

$$
\dot{g}=\Xi(g, u), \quad g \in \mathrm{G}, u \in U
$$

where $\Xi(g, u)=g \Xi(\mathbf{1}, u) \in T_{g} \mathrm{G}$. (The notation $g \Xi(\mathbf{1}, u)$ stands for the image of the element $\Xi(\mathbf{1}, u) \in \mathfrak{g}$ under the tangent map of the left translation $d L_{g}=T_{1} L_{g}: \mathfrak{g} \rightarrow T_{g} \mathrm{G}$.) For the purposes of this paper, we assume that $U$ is a smooth manifold (of dimension $\ell$ ). Also, admissible controls will be piecewise continuous $U$-valued maps, defined on compact intervals $[0, T]$. Note that the 
family $X=\left(\Xi_{u}=\Xi(\cdot, u)\right)_{u \in U}$ consists of left-invariant vector fields on G. To avoid degeneracies, we further assume that the (regular) parametrisation map $\Xi(\mathbf{1}, \cdot): U \rightarrow \mathfrak{g}$ is an embedding. Since submanifolds are precisely the images of embeddings, the condition above says, equivalently, that the image set $\Gamma=$ $\operatorname{im} \Xi(\mathbf{1}, \cdot) \subseteq \mathfrak{g}$ is a submanifold of $\mathfrak{g}$. We shall refer to this (parametrised) submanifold as the trace of $\Sigma$. By identifying (the left-invariant vector field) $\Xi(\cdot, u) \in \mathfrak{X}^{L}(\mathrm{G})$ with $\Xi(\mathbf{1}, u) \in \mathfrak{g}$, we have that $\Gamma=\left\{\Xi_{u} \mid u \in U\right\}$.

A trajectory for an admissible control $u(\cdot):[0, T] \rightarrow U$ is an absolutely continuous curve $g(\cdot):[0, T] \rightarrow \mathrm{G}$ such that $\dot{g}(t)=g(t) \Xi(\mathbf{1}, u(t))$ for almost every $t \in[0, T]$. The Carathéodory existence and uniqueness theorem of ordinary differential equations implies the local existence and global uniqueness of trajectories. A remarkable property of left-invariant systems is that a left translation of a trajectory is a trajectory.

The attainable set (from the identity $\mathbf{1} \in \mathrm{G}$ ) is the set $\mathcal{A}$ of all terminal points $g(T)$ of all trajectories $g(\cdot):[0, T] \rightarrow \mathrm{G}$ starting at $\mathbf{1}$. We say that a system $\Sigma$ is controllable if for any $g_{0}, g_{1} \in \mathrm{G}$, there exists a $T \geq 0$ and a trajectory $g(\cdot):[0, T] \rightarrow \mathrm{G}$ such that $g(0)=g_{0}$ and $g(T)=g_{1}$. A system is controllable if and only if $\mathcal{A}=\mathrm{G}$. Necessary conditions for controllability are that the group $\mathrm{G}$ be connected and that the Lie algebra Lie $(\Gamma)$ generated by the trace $\Gamma \subseteq \mathfrak{g}$ be the whole space $\mathfrak{g}$.

The orbit (through the identity $\mathbf{1}$ ) is the unique connected virtual Lie subgroup $\mathcal{O}$ of the state space $G$ with Lie algebra Lie $(\Gamma)$ (cf. [4]). We then have that $\mathcal{A} \subseteq \mathcal{O}$. An orbit may be described as the smallest connected virtual Lie subgroup containing the attainable set. We have the following characterisation of points in the orbit.

Proposition 1. Let $\Sigma$ be a system with orbit $\mathcal{O}$. Then $g \in \mathcal{O}$ if and only if there exists a piecewise constant function $v(\cdot):[0, T] \rightarrow\{-1,1\}$, an admissible control $u(\cdot):[0, T] \rightarrow U$ and an absolutely continuous mapping $g(\cdot):[0, T] \rightarrow$ $\mathrm{G}$ such that $g(0)=\mathbf{1}, g(T)=g$ and

$$
\dot{g}(t)=v(t) \Xi(g(t), u(t))
$$

for almost every $t \in[0, T]$.

This follows as the collection of endpoints $g(T)$ form a path connected subgroup of $\mathrm{G}$ and as such is the unique connect virtual Lie subgroup of $\mathrm{G}$ with Lie algebra Lie $(\Gamma)$, namely $\mathcal{O}$. Closure of the group product is proven by concatenation, and inversion by reverse traversing curves. 


\section{The category LiCS}

We define now the concrete category LiCS of left-invariant control systems. An object in LiCS is a pair $\Sigma=(\mathrm{G}, \Xi)$, where the state space $\mathrm{G}$ is a (real, finite-dimensional) Lie group and the dynamics $\Xi: \mathrm{G} \times U \rightarrow T \mathrm{G}$ is leftinvariant; that is, for any $g \in \mathrm{G}$, the diagram

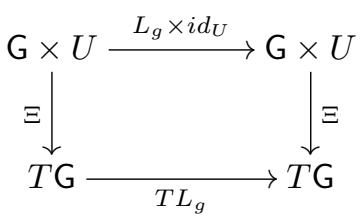

commutes. This means that, for any $g \in \mathrm{G}$ and any $u \in U, \Xi(g, u)=$ $g \Xi(\mathbf{1}, u)$. The map $\Xi(\mathbf{1}, \cdot): U \rightarrow \mathfrak{g}$ is a (smooth) embedding (hence, the trace $\Gamma$ is a submanifold of the Lie algebra $\mathfrak{g})$. A morphism $\Phi=(\phi, \varphi): \Sigma \rightarrow \Sigma^{\prime}$ in $\mathbf{L i C S}$ is a mapping

$$
\Phi: \mathrm{G} \times U \rightarrow \mathrm{G}^{\prime} \times U^{\prime}, \quad(g, u) \mapsto(\phi(g), \varphi(g, u))
$$

where the state component $\phi: \mathrm{G} \rightarrow \mathrm{G}^{\prime}$ and the feedback component $\varphi$ : $\mathrm{G} \times U \rightarrow U^{\prime}$ are smooth maps such that the diagram

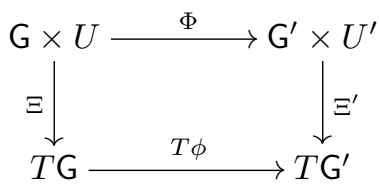

commutes. The "invariance condition" is equivalent to the condition that, for any LiCS-object $\Sigma=(\mathrm{G}, \Xi)$ and any $g \in \mathrm{G}, L_{g} \times \mathrm{id}_{U}$ is a LiCS-morphism.

Remark. The category LiCS may be viewed as an "extension" of the category LGrp of (real, finite dimensional) Lie groups (in the sense that it has a subcategory functorially isomorphic to it).

Henceforth, we shall refer to a LiCS-object simply as a system and to a LiCS-morphism as a morphism. We shall customarily call the surjective morphisms, epimorphisms and the bijective morhisms, bimorphisms. For a morphism $\Phi=(\phi, \varphi)$, we will say that (the feedback component) $\varphi$ is Ginvariant if it does not depend on $G$ explicitly.

We say that a system $\Sigma=(\mathrm{G}, \Xi)$ is connected if its state space $\mathrm{G}$ is connected. Also, such a system has full rank if its trace generates the Lie algebra $\mathfrak{g}$ (i.e., Lie $(\Gamma)=\mathfrak{g})$. 
Remark. The left-invariant control affine systems form a full subcategory of LiCS, denoted by LiCAS. A LiCAS-object is a system $\Sigma=(\mathrm{G}, \Xi)$ whose parametrisation map is affine:

$$
\Xi(\mathbf{1}, \cdot): \mathbb{R}^{\ell} \rightarrow \mathfrak{g}, \quad u \mapsto A+u_{1} B_{1}+\cdots+u_{\ell} B_{\ell} .
$$

Any system with an affine trace is isomorphic to a LiCAS-object by means of a reparametrisation of the trace (see proposition 6). A pertinent specialisation is that the feedback component of a morphism in LiCAS be affine (cf. [15]). This means that, if $\Phi=(\phi, \varphi): \Sigma \rightarrow \Sigma^{\prime}$ is a morphism in LiCAS, then

$$
\varphi(g, u)=\varphi(g, 0)+\Lambda(g) \cdot u
$$

where (for each $g \in G) \Lambda(g) \in \mathrm{L}\left(\mathbb{R}^{\ell}, \mathbb{R}^{\ell^{\prime}}\right)$.

We shall investigate some basic properties of the morphisms. We start with a few observations, collected up in the next proposition. The proof is easy and will be omitted.

Proposition 2. Let $\Sigma$ and $\Sigma^{\prime}$ be two systems.

(i) If $\Phi=(\phi, \varphi): \Sigma \rightarrow \Sigma^{\prime}$ is a morphism, then (the feedback component) $\varphi$ is uniquely determined by

$$
\Xi^{\prime}(\mathbf{1}, \varphi(g, u))=T_{\mathbf{1}}\left(L_{(\phi(g))^{-1}} \circ \phi \circ L_{g}\right) \cdot \Xi(\mathbf{1}, u) .
$$

(ii) If $\Phi, \Phi^{\prime}: \Sigma \rightarrow \Sigma^{\prime}$ are morphisms, then $\Phi=\Phi^{\prime}$ if and only if $\phi=\phi^{\prime}$.

(iii) If $\phi: \mathrm{G} \rightarrow \mathrm{G}^{\prime}$ is a smooth map such that (for all $g \in \mathrm{G}$ )

$$
T_{1}\left(L_{(\phi(g))^{-1}} \circ \phi \circ L_{g}\right) \cdot \Gamma \subseteq \Gamma^{\prime}
$$

then there exists a (unique) morphism $\Phi=(\phi, \varphi): \Sigma \rightarrow \Sigma^{\prime}$.

Propagating trajectories from a system to another is a desirable feature since most properties of control systems are (or, at least, can be related to) properties of their trajectories. The morphisms in LiCS are precisely those based on trajectory propagating maps.

Proposition 3. Let $\Sigma=(\mathrm{G}, \Xi)$ and $\Sigma^{\prime}=\left(\mathrm{G}^{\prime}, \Xi^{\prime}\right)$ be two systems and let $\phi: \mathrm{G} \rightarrow \mathrm{G}^{\prime}$ be a smooth map. Then there exists a (unique) morphism $\Phi=$ $(\phi, \varphi): \Sigma \rightarrow \Sigma^{\prime}$ if and only if $\phi$ maps trajectories of $\Sigma$ to trajectories of $\Sigma^{\prime}$.

Proof. Assume $\Phi=(\phi, \varphi): \Sigma \rightarrow \Sigma^{\prime}$ is a morphism. Let $g(\cdot):[0, T] \rightarrow \mathrm{G}$ be a trajectory of $\Sigma$. Then $\phi \circ g(\cdot):[0, T] \rightarrow \mathrm{G}^{\prime}$ is a trajectory of $\Sigma^{\prime}$. Indeed, we have that (almost everywhere)

$$
\frac{d}{d t} \phi(g(t))=T_{g(t)} \phi \cdot \dot{g}(t)=T_{g(t)} \phi \cdot \Xi(g(t), u(t))=\Xi^{\prime}(\phi(g(t)), \varphi(g(t), u(t))) .
$$


Since the map $t \mapsto \varphi(g(t), u(t)):[0, T] \rightarrow U^{\prime}$ is piecewise continuous, it is an admissible control of $\Sigma^{\prime}$ and hence $\phi \circ g(\cdot)$ is a trajectory of $\Sigma^{\prime}$.

For the converse, assume that $\phi$ maps each trajectory of $\Sigma$ to a trajectory of $\Sigma^{\prime}$. Let $g \in \mathrm{G}, u \in U$ and $g(\cdot):[0, T] \rightarrow \mathrm{G}$ be the trajectory of $\Sigma$ defined by $g(0)=g$ and $\dot{g}(t)=\Xi(g(t), u)$. Then $\phi \circ g(\cdot)$ is a trajectory of $\Sigma$ and hence there exists a (piecewise continuous) control $w(\cdot):[0, T] \rightarrow U^{\prime}$ such that $\frac{d}{d t} \phi(g(t))=\Xi^{\prime}(\phi(g(t)), w(t))$ implying that $T_{\mathbf{1}}\left(L_{(\phi(g))^{-1}} \circ \phi \circ L_{g}\right) \cdot \Xi(\mathbf{1}, u) \in$ $\Gamma^{\prime}$. Thus

$$
T_{1}\left(L_{(\phi(g))^{-1}} \circ \phi \circ L_{g}\right) \cdot \Gamma \subseteq \Gamma^{\prime}
$$

and hence (by proposition 2), there exists a (unique) morphism $\Phi$ with $\phi$ as state component.

Remark. The above characterisation of trajectory propagating maps is similar to the one presented in [11] for a class of partially more general control systems.

Corollary. Let $\Phi=(\phi, \varphi): \Sigma \rightarrow \Sigma^{\prime}$ be a morphism. Then $\phi(\mathcal{A}) \subseteq \phi(\mathbf{1}) \mathcal{A}^{\prime}$.

Corollary. Let $\Phi=(\phi, \varphi): \Sigma \rightarrow \Sigma^{\prime}$ be a morphism with (the state space component) $\phi$ surjective. If $\Sigma$ is controllable, then so is $\Sigma^{\prime}$. (Equivalently, if $\Sigma^{\prime}$ is not controllable, then neither is $\Sigma$.)

Of particular interest in the study of (left-invariant control) systems are diffeomorphisms between the state spaces of the two systems that preserve left-invariant vector fields. As such, it is of interest to characterise those morphisms for which the push forward of a left-invariant vector field (by the state component of the morphism) is also left-invariant.

Proposition 4. Let $\Phi=(\phi, \varphi): \Sigma \rightarrow \Sigma^{\prime}$ be a morphism such that (the state component) $\phi$ is a diffeomorphism. Then the push forward $\phi_{*} \Xi_{u}$ of a leftinvariant vector field $\Xi_{u}$ of $\Sigma$ is a left-invariant vector field on $\Sigma^{\prime}$ if and only if (the feedback component) $\varphi$ is $\mathrm{G}$-invariant.

Proof. Let $u \in U$ and $g \in \mathrm{G}$. We have (by the definition of the push forward)

$$
\left(\phi_{*} \Xi_{u}\right)(\phi(g))=T_{g} \phi \cdot \Xi(g, u)=\Xi^{\prime}(\phi(g), \varphi(g, u)) .
$$

Then $\left(\phi_{*} \Xi_{u}\right)(\mathbf{1})=B_{u} \in \Gamma^{\prime}$. Assuming that $\phi_{*} \Xi_{u}$ is left invariant, we have that $\left(\phi_{*} \Xi_{u}\right)(\phi(g))=T_{\mathbf{1}} L_{\phi(g)} \cdot B_{u}$. Thus $\Xi^{\prime}(\mathbf{1}, \varphi(g, u))=B_{u}$ for all $g \in \mathrm{G}$. As $\Xi^{\prime}(\mathbf{1}, \cdot)$ is injective, the result follows.

Conversely, assuming $\varphi$ is G-invariant, we have that $\left(\phi_{*} \Xi_{u}\right)(\phi(g))=$ $T_{\mathbf{1}} L_{\phi(g)} \cdot \Xi^{\prime}(\mathbf{1}, \varphi(\mathbf{1}, u))$. Thus $\phi_{*} \Xi_{u}=\Xi_{\varphi(\mathbf{1}, u)}^{\prime}$ is a left-invariant vector field. 
Remark. The category LiCS is not balanced (i.e., not every bimorphism is an isomorphism). Indeed, consider the system $\Sigma=(\mathbb{R}, \Xi)$, where

$$
\Xi(\mathbf{1}, \cdot): \mathbb{R}^{0}=\{0\} \rightarrow \mathbb{R}, \quad 0 \mapsto 0 \quad(\text { here } \mathbf{1}=0 \in \mathbb{R}) .
$$

Then $(\phi, \varphi): \Sigma \rightarrow \Sigma, \quad(x, 0) \mapsto\left(x^{3}, 0\right)$ is a bimorphism, but not an isomorphism (as $x \mapsto x^{3}$ is not a diffeomorphism).

We do have the following characterisation of isomorphisms in $\mathbf{L i C S}$.

Proposition 5. A morphism $\Phi=(\phi, \varphi): \Sigma \rightarrow \Sigma^{\prime}$ is an isomorphism if and only if it is a bimorphism and (the state component) $\phi$ has full rank.

Proof. One implication is clearly trivial. For the converse, assume that $\Phi=$ $(\phi, \varphi)$ is a bimorphism and that $\phi$ has full rank. Then it follows that $\phi: G \rightarrow$ $\mathrm{G}^{\prime}$ is a diffeomorphism. As $\Phi$ is an epimorphism, we have that (for $g \in \mathrm{G}$ )

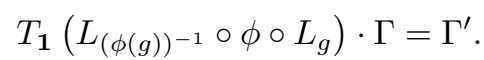

Hence, we have that (for $g^{\prime} \in \mathrm{G}^{\prime}$ )

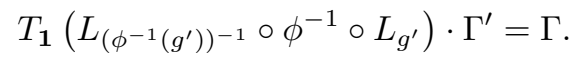

Thus there exists a (unique) morphism $\Phi^{\prime}: \Sigma^{\prime} \rightarrow \Sigma$ with $\phi^{-1}$ as state component (see proposition 2). It is easy to verify that $\Phi^{\prime} \circ \Phi=i d_{\Sigma}$ and $\Phi \circ \Phi^{\prime}=i d_{\Sigma^{\prime}}$.

Next we show that the reparametrisation of the trace of a system yields an isomorphic system.

Proposition 6. Let $\Sigma$ and $\Sigma^{\prime}$ be two systems with the same state space $\mathrm{G}$ and the same trace $\Gamma \subseteq \mathfrak{g}$. Then there exists an isomorphism $\Phi: \Sigma \rightarrow$ $\Sigma^{\prime},(g, u) \mapsto(g, \bar{\varphi}(u))$, where $\bar{\varphi}: U \rightarrow U^{\prime}$ is a diffeomorphism.

Proof. As $\Xi(\mathbf{1}, \cdot): U \rightarrow \Gamma$ and $\Xi^{\prime}(\mathbf{1}, \cdot): U^{\prime} \rightarrow \Gamma$ are diffeomorphisms, we define $\bar{\varphi}$ to be the (unique) diffeomorphism such that $\Xi(\mathbf{1}, \cdot)=\Xi^{\prime}(\mathbf{1}, \bar{\varphi}(\cdot))$. Then we have that $T_{1} i d_{\mathrm{G}} \cdot \Xi(g, u)=\Xi^{\prime}(g, \bar{\varphi}(u))$ and so (by proposition 5 ) $i d_{\mathrm{G}} \times \bar{\varphi}$ is the required isomorphism.

To conclude this section, we investigate the relationship between morphisms in LGrp (i.e., Lie group homomorphisms) and morphisms in LiCS.

Proposition 7. Let $\Sigma$ and $\Sigma^{\prime}$ be two systems.

(i) If $\phi: \mathrm{G} \rightarrow \mathrm{G}^{\prime}$ is a Lie group homomorphism such that $T_{\mathbf{1}} \phi \cdot \Gamma \subseteq \Gamma^{\prime}$, then there exists a unique morphism $\Phi=(\phi, \varphi): \Sigma \rightarrow \Sigma^{\prime}$ such that (the feedback component) $\varphi$ is G-invariant. 
(ii) If $\Phi=(\phi, \varphi): \Sigma \rightarrow \Sigma^{\prime}$ is a morphism such that $\phi(\mathbf{1})=\mathbf{1}$ and (the feedback component) $\varphi$ is $\mathrm{G}$-invariant, then $\left.\phi\right|_{\mathcal{O}}: \mathcal{O} \rightarrow \mathrm{G}^{\prime}$ is a Lie group homomorphism such that $\operatorname{im}\left(\left.\phi\right|_{\mathcal{O}}\right) \subseteq \mathcal{O}^{\prime}$.

Proof. (i) We have that $L_{(\phi(g))^{-1}} \circ \phi \circ L_{g}=\phi$, thus (for $g \in \mathrm{G}, u \in U$ )

$$
T_{\mathbf{1}}\left(L_{(\phi(g))^{-1}} \circ \phi \circ L_{g}\right) \cdot \Gamma=T_{\mathbf{1}} \phi \cdot \Gamma \subseteq \Gamma^{\prime} .
$$

Then (by proposition 2) there exists a unique morphism $(\phi, \varphi): \Sigma \rightarrow \Sigma^{\prime}$ such that $\Xi^{\prime}(\mathbf{1}, \varphi(g, u))=T_{\mathbf{1}} \phi \cdot \Xi(\mathbf{1}, u)$. Hence, $\varphi$ is G-invariant.

(ii) As $\mathcal{O}$ is an immersed submanifold of $G$, we have that $\left.\phi\right|_{\mathcal{O}}$ is smooth. Let $g_{1}, g_{2} \in \mathcal{O}$. Then there exist $T_{i}>0, v_{i}(\cdot):\left[0, T_{i}\right] \rightarrow\{-1,1\}, u_{i}(\cdot)$ : $\left[0, T_{i}\right] \rightarrow U$ and $g_{i}(\cdot):\left[0, T_{i}\right] \rightarrow \mathrm{G}, i=1,2$ as prescribed in proposition 1.

Hence, we have (almost everywhere) that

$\frac{d}{d t} \phi\left(g_{1}(t)\right)=T_{g_{1}(t)} \phi \cdot v_{1}(t) \Xi\left(g_{1}(t), u_{1}(t)\right)=v_{1}(t) \Xi^{\prime}\left(\phi\left(g_{1}(t)\right), \varphi\left(g_{1}(t), u_{1}(t)\right)\right)$.

Since $t \mapsto \varphi\left(g_{1}(t), u_{1}(t)\right)$ is an admissible control, it follows that $\phi\left(g_{1}\right) \in \mathcal{O}^{\prime}$ (by proposition 1) and so $\operatorname{im}\left(\left.\phi\right|_{\mathcal{O}}\right) \subseteq \mathcal{O}^{\prime}$. We are left to show that $\left.\phi\right|_{\mathcal{O}}$ is a group homomorphism. Consider the (concatenated) curves

$$
\begin{aligned}
& c_{1}(t)= \begin{cases}g_{1}(t) & 0 \leq t \leq T_{1} \\
g_{1} g_{2}\left(t-T_{1}\right) & T_{1}<t \leq T_{1}+T_{2}\end{cases} \\
& c_{2}(t)= \begin{cases}\phi\left(g_{1}(t)\right) & 0 \leq t \leq T_{1} \\
\phi\left(g_{1}\right) \phi\left(g_{2}\left(t-T_{1}\right)\right) & T_{1}<t \leq T_{1}+T_{2} .\end{cases}
\end{aligned}
$$

Then, as $\varphi$ is G-invariant, we have (almost everywhere) that

$$
\begin{aligned}
\frac{d}{d t} \phi\left(c_{1}(t)\right) & = \begin{cases}v_{1}(t) \Xi^{\prime}\left(\phi\left(c_{1}(t)\right), \varphi\left(\mathbf{1}, u_{1}(t)\right)\right) & 0 \leq t \leq T_{1} \\
v_{2}(t) \Xi^{\prime}\left(\phi\left(c_{1}(t)\right), \varphi\left(\mathbf{1}, u_{2}\left(t-T_{1}\right)\right)\right) & T_{1}<t \leq T_{1}+T_{2}\end{cases} \\
\frac{d}{d t} c_{2}(t) & = \begin{cases}v_{1}(t) \Xi^{\prime}\left(c_{2}(t), \varphi\left(\mathbf{1}, u_{1}(t)\right)\right) & 0 \leq t \leq T_{1} \\
v_{2}(t) \Xi^{\prime}\left(c_{2}(t), \varphi\left(\mathbf{1}, u_{2}\left(t-T_{1}\right)\right)\right) & T_{1}<t \leq T_{1}+T_{2} .\end{cases}
\end{aligned}
$$

Thus, as $\phi \circ c_{1}(\cdot)$ and $c_{2}(\cdot)$ solve the same Cauchy problem, we get that they are equal. Hence $\phi\left(g_{1} g_{2}\right)=\phi\left(c_{1}\left(T_{1}+T_{2}\right)\right)=c_{2}\left(T_{1}+T_{2}\right)=\phi\left(g_{1}\right) \phi\left(g_{2}\right)$.

Corollary. Let $\Sigma$ and $\Sigma^{\prime}$ be full-rank connected systems and $\phi: G \rightarrow \mathrm{G}^{\prime}$ be a smooth map such that $\phi(\mathbf{1})=\mathbf{1}$. Then there exists a unique morphism $\Phi=(\phi, \varphi): \Sigma \rightarrow \Sigma^{\prime}$ such that (the feedback component) $\varphi$ is G-invariant if and only if $\phi$ is a Lie group homomorphism such that $T_{\mathbf{1}} \phi \cdot \Gamma \subseteq \Gamma^{\prime}$. 


\section{Covering systems}

We say that a connected system $\Sigma^{\prime}$ covers a connected system $\Sigma$ if there exists an epimorphism $\Theta=(\theta, \vartheta): \Sigma^{\prime} \rightarrow \Sigma$ such that (the state component) $\theta: \mathrm{G}^{\prime} \rightarrow \mathrm{G}$ is a Lie group epimorphism with discrete kernel. Such a morphism is called a covering morphism and the pair $\left(\Sigma^{\prime}, \Theta\right)$ will be referred to as a covering of $\Sigma$. Let us note that, for a covering morphism $\Theta=(\theta, \vartheta)$, the feedback component $\vartheta$ is $\mathrm{G}^{\prime}$-invariant (by proposition 7 ) and $T_{\mathbf{1}} \theta \cdot \Gamma^{\prime}=\Gamma$.

Remark. The pair $(\Theta, \Sigma)$ is a quotient object of $\Sigma^{\prime}$ in LiCS.

Coverings can be viewed as objects of a comma category. We form a subcategory of LiCS containing only connected systems and only covering morphisms as morphisms; we denote the inclusion functor for this subcategory by $\mathcal{J}$. The comma category $(\mathcal{J} \downarrow \boldsymbol{\Sigma})$ then has coverings of $\Sigma$ as objects. Morphisms $\Theta:\left(\Sigma_{1}^{\prime}, \Theta_{1}\right) \rightarrow\left(\Sigma_{2}^{\prime}, \Theta_{2}\right)$ in $(\mathcal{J} \downarrow \boldsymbol{\Sigma})$ are covering morphisms $\Theta: \Sigma_{1}^{\prime} \rightarrow \Sigma_{2}^{\prime}$ such that $\Theta_{1}=\Theta_{2} \circ \Theta$ (cf. [10]).

Given a connected system $\Sigma=(\mathrm{G}, \Xi)$ and a Lie group covering homomorphism $\theta: G^{\prime} \rightarrow G$ (with $G^{\prime}$ connected), a covering $\left(\Sigma^{\prime}, \Theta\right)$ of $\Sigma$ such that $\theta$ is the state component of $\Theta$, can be constructed as follows:

$$
\begin{gathered}
\Xi^{\prime}: \mathrm{G}^{\prime} \times U \rightarrow T \mathrm{G}^{\prime}, \quad\left(g^{\prime}, u\right) \mapsto\left(T_{g^{\prime}} \theta\right)^{-1} \cdot \Xi\left(\theta\left(g^{\prime}\right), u\right) \\
\Theta: \mathrm{G}^{\prime} \times U \rightarrow \mathrm{G} \times U, \quad\left(g^{\prime}, u\right) \mapsto\left(\theta\left(g^{\prime}\right), u\right) .
\end{gathered}
$$

We say that a covering $(\widetilde{\Sigma}, \Theta)$ of a connected system $\Sigma$ is a universal covering if the state space $\widetilde{G}$ is simply connected. For any connected system $\Sigma$ there is a (universal) Lie group covering homomorphism $\theta: \widetilde{G} \rightarrow G$. Thus we can always construct a covering $(\widetilde{\Sigma}, \Theta)$ of a connected system $\Sigma$ such that the state space $\widetilde{G}$ is simply connected. A universal covering $(\widetilde{\Sigma}, \Theta)$ may be viewed as an initial object of the comma category $(\mathcal{J} \downarrow \boldsymbol{\Sigma})$. Thus, in particular, the category $(\mathcal{J} \downarrow \boldsymbol{\Sigma})$ has initial objects.

Proposition 8. A universal covering $(\widetilde{\Sigma}, \Theta)$ of a connected system $\Sigma$ is an initial object in the comma category $(\mathcal{J} \downarrow \mathbf{\Sigma})$.

Proof. Suppose $\left(\widetilde{\Sigma}, \Theta_{1}\right)$ is a universal covering of a connected system $\Sigma$. We need to show that for any object $\left(\Sigma^{\prime}, \Theta_{2}\right)$ in $(\mathcal{J} \downarrow \Sigma)$, there exists a unique morphism $\Theta:\left(\widetilde{\Sigma}, \Theta_{1}\right) \rightarrow\left(\Sigma^{\prime}, \Theta_{2}\right)$ in $(\mathcal{J} \downarrow \boldsymbol{\Sigma})$. We have an isomorphism of Lie algebras $\left(T_{\mathbf{1}} \theta_{2}\right)^{-1} \cdot T_{\mathbf{1}} \theta_{1}: \tilde{\mathfrak{g}} \rightarrow \mathfrak{g}^{\prime}$. As $\widetilde{\mathrm{G}}$ is simply connected, there exists a unique Lie group epimorphism $\theta: \widetilde{G} \rightarrow G^{\prime}$ with discrete kernel and tangent map $\left(T_{\mathbf{1}} \theta_{2}\right)^{-1} \cdot T_{\mathbf{1}} \theta_{1}$ at identity (see [4], [8]). Now $T_{\mathbf{1}} \theta \cdot \tilde{\Gamma}=\Gamma^{\prime}$, thus there exists (by proposition 7) a unique covering morphism $\Theta=(\theta, \vartheta): \widetilde{\Sigma} \rightarrow \Sigma^{\prime}$. As $\theta_{1}=\theta_{2} \circ \theta$ by the construction of $\theta$, (by proposition 2) we get that $\Theta_{1}=\Theta_{2} \circ \Theta$. 
As initial objects of a category are unique up to isomorphism, we have the following corollary.

Corollary. A universal covering $(\widetilde{\Sigma}, \Theta)$ of a connected system $\Sigma$ is unique up to an isomorphism in $(\mathcal{J} \downarrow \mathbf{\Sigma})$.

Universal coverings (of connected systems) satisfy the following universal property.

Proposition 9. Let $\Sigma$ and $\Sigma^{\prime}$ be two systems. Then, given a morphism $\Phi$ : $\Sigma \rightarrow \Sigma^{\prime}$, universal coverings $(\widetilde{\Sigma}, \Theta)$ and $\left(\widetilde{\Sigma}^{\prime}, \Theta^{\prime}\right)$ of $\Sigma$ and $\Sigma^{\prime}$, respectively, and elements $\tilde{g} \in \widetilde{\mathrm{G}}, \tilde{g}^{\prime} \in \widetilde{\mathrm{G}}^{\prime}$ such that $\phi(\theta(\tilde{g}))=\theta^{\prime}\left(\tilde{g}^{\prime}\right)$, there exists a unique covering morphism $\widetilde{\Phi}: \widetilde{\Sigma} \rightarrow \widetilde{\Sigma}^{\prime}$ such that $\tilde{\phi}(\tilde{g})=\tilde{g}^{\prime}$ and the diagram

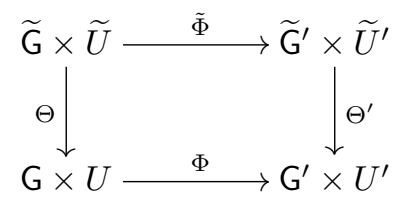

commutes.

Proof. The functorial property of a universal covering for (connected) smooth manifolds (cf. [4], [8]) implies the existence of a unique smooth map $\tilde{\phi}: \widetilde{G} \rightarrow$ $\widetilde{\mathrm{G}}^{\prime}$ such that $\tilde{\phi}(\tilde{g})=\tilde{g}^{\prime}$ and $\theta^{\prime} \circ \tilde{\phi}=\phi \circ \theta$. Hence, we have (for $\tilde{g} \in \widetilde{\mathrm{G}}$ )

$$
T_{\tilde{g}} \tilde{\phi} \cdot\left(T_{\tilde{g}} \theta\right)^{-1} \cdot T_{\mathbf{1}} L_{\theta(\tilde{g})} \cdot \Gamma=\left(T_{\tilde{\phi}(\tilde{g})} \theta^{\prime}\right)^{-1} \cdot T_{\theta(\tilde{g})} \phi \cdot T_{\mathbf{1}} L_{\theta(\tilde{g})} \cdot \Gamma .
$$

This implies

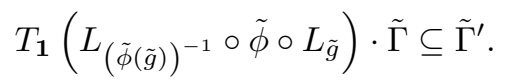

Thus (by proposition 2), there exists a unique morphism $\widetilde{\Phi}=(\tilde{\phi}, \tilde{\varphi}): \widetilde{\Sigma} \rightarrow \widetilde{\Sigma}^{\prime}$ and $\Theta^{\prime} \circ \widetilde{\Phi}=\Phi \circ \Theta$ (as the corresponding state components are identical).

Proposition 10. If $\left(\Sigma^{\prime}, \Theta\right)$ is a covering of a connected system $\Sigma$, then $\theta\left(\mathcal{A}^{\prime}\right)=\mathcal{A}$.

Proof. By the (first) corollary to proposition 3, we have that $\theta\left(\mathcal{A}^{\prime}\right) \subseteq \mathcal{A}$. For $g \in \mathcal{A}$, there exists a trajectory $g(\cdot):[0, T] \rightarrow \mathrm{G}$ with admissible control $u(\cdot):[0, T] \rightarrow U$ such that $g(0)=\mathbf{1}, g(T)=g$ and $\dot{g}(t)=g(t) \Xi(\mathbf{1}, u(t))$ almost everywhere. Let $u^{\prime}(\cdot):[0, T] \rightarrow U^{\prime}$ be defined by

$$
\Xi^{\prime}\left(\mathbf{1}, u^{\prime}(t)\right)=\left(T_{\mathbf{1}} \theta\right)^{-1} \cdot \Xi(\mathbf{1}, u(t)) .
$$


Then $u^{\prime}(\cdot)$ is an admissible control and the Cauchy problem

$$
\dot{g}^{\prime}(t)=g^{\prime}(t) \Xi^{\prime}\left(\mathbf{1}, u^{\prime}(t)\right) \text { and } \quad g^{\prime}(0)=\mathbf{1}
$$

has a unique solution $g^{\prime}(\cdot):[0, T] \rightarrow \mathrm{G}^{\prime}$. Then $g^{\prime}(\cdot)$ is a trajectory of $\Sigma^{\prime}$ and, as $\left.T_{\mathbf{1}} \theta \cdot \Xi^{\prime}\left(\mathbf{1}, u^{\prime}(t)\right)=\Xi(\mathbf{1}, u(t))\right)$, the two trajectories $\phi \circ g^{\prime}(\cdot)$ and $g(\cdot)$ solve the same Cauchy problem. So we get $\theta \circ g^{\prime}(\cdot)=g(\cdot)$. Hence, as $g^{\prime}(T) \in \mathcal{A}^{\prime}$ and $\theta\left(g^{\prime}(T)\right)=g$, we conclude that $\mathcal{A} \subseteq \theta\left(\mathcal{A}^{\prime}\right)$.

\section{Final remarks}

In the study of (left-invariant) control systems, it is natural to restrict ourselves to controllable systems and transformations of such systems preserving left-invariant vector fields. We have that controllable systems are necessarily full-rank connected systems and that a morphism $\Phi=(\phi, \varphi)$ (between such systems), preserving left-invariant vector fields, is exactly one whose feedback component is G-invariant. But this, in turn, is equivalent to the state component $\phi$ being a Lie group homomorphism such that $T_{\mathbf{1}} \phi \cdot \Gamma \subseteq \Gamma^{\prime}$. This provides a simple algebraic characterisation of the morphisms of interest.

With the basic structure of LiCS in place, it is of interest to study the (state space and feedback) equivalence of such systems. The discussion above indicates that these equivalences can be specialised and algebraically characterised in LiCS. These characterisations would then create the possibility of effectively classifying systems of a certain class (for example, low dimensional LiCAS-objects). In this setting, covering morphisms would serve to relate locally equivalent systems on different Lie groups, but with the same Lie algebra. All these are topics for future research.

\section{References}

[1] A.A. Agrachev and Y.L. Sachkov, Control Theory from the Geometric Viewpoint, Springer-Verlag, Berlin, 2004.

[2] R.W. Brockett, System theory on group manifolds and coset spaces, SIAM J. Control 10(2)(1972), 265-284.

[3] V.I. Elkin, Affine control systems: their equivalence, classification, quotient systems and subsystems, J. Math. Sci. 88(5)(1998), 675-721.

[4] V.V. Gorbatsevich, A.L. Onishchik and E.B. Vinberg, Foundations of Lie Theory and Lie Transformation Groups, Springer-Verlag, Berlin, 1997. 
[5] E. Haghverdi, P. Tabuada and G. Pappas, Bisimulation relations for dynamical and control systems, Electron. Notes Theor. Comp. Sci. 69(2003), 120-136.

[6] V. Jurdjevic, Geometric Control Theory, Cambridge University Press, Cambridge, 1997.

[7] V. Jurdjevic and H.J. Sussmann, Control systems on Lie groups, J. Diff. Equations 12(1972), 313-329.

[8] A.W. Knapp, Lie Groups Beyond an Introduction (Second Edition), Birkhäuser, Boston, 2004.

[9] A.D. Lewis, The Category of Affine Connection Control Systems, in Proc. 39th IEEE Conf. Decision \& Control, Sydney, Australia, 2000, pp. 12601265.

[10] S. Mac Lane, Categories for the Working Mathematician (Second Edition), Springer-Verlag, New York, 1997.

[11] G.J. Pappas, G. Lafferriere and S. Sastry, Hierarchically consistent control systems, IEEE Trans. Automat. Control 45(6)(2000), 1144-1160.

[12] C.C. Remsing, Optimal control and Hamilton-Poisson formalism, Int. J. Pure Appl. Math. 59(1)(2010), 11-17.

[13] Y.L. Sachkov, Control theory on Lie groups, J. Math. Sci. 156(3)(2009), 381-439.

[14] H.J. Sussmann, Lie brackets, real analyticity and geometric control, in Differential Geometric Control Theory (R.W. Brockett, R.S. Millman, H.J. Sussmann, eds.), Birkhäuser, 1983, pp. 1-116.

[15] P. Tabuada, On the Factorization of Trajectory Lifting Maps, in Proc. 44th IEEE Conf. Decision \& Control, Seville, Spain, 2005, pp. 4225-4230.

[16] P. Tabuada and G.J. Pappas, Bisimilar control affine systems, Syst. Control Lett. 52(1)(2004), 49-58.

[17] P. Tabuada and G.J. Pappas, Quotients of fully nonlinear control systems, SIAM J. Control Optim. 43(5)(2005), 1844-1866.

Rory BIGGS,

Department of Mathematics (Pure and Applied),

Rhodes University,

PO Box 94, 6140 Grahamstown, South Africa.

Email: rorybiggs@gmail.com 
Claudiu C. REMSING,

Department of Mathematics (Pure and Applied),

Rhodes University,

PO Box 94, 6140 Grahamstown, South Africa.

Email: c.c.remsing@ru.ac.za 
